\title{
Universal aspects of Efimov states and light halo nuclei
}

\author{
A. E. A. Amorim, ${ }^{1,2}$ T. Frederico,${ }^{3}$ and Lauro Tomio ${ }^{1}$ \\ ${ }^{1}$ Instituto de Física Teórica, Universidade Estadual Paulista, 01405-900 São Paulo, Brazil \\ ${ }^{2}$ Faculdade de Tecnologia de Jahu, CEETEPS, Jahu, Brazil \\ ${ }^{3}$ Departamento de Física, Instituto Tecnológico de Aeronáutica, Centro Técnico Aeroespacial, \\ 12228-900 São José dos Campos, Brazil
}

(Received 16 December 1996)

\begin{abstract}
The parametric region in the plane defined by the ratios of the energies of the subsystems and the three-body ground state, in which Efimov states can exist, is determined. We use a renormalizable model that guarantees the general validity of our results in the context of short-range interactions. The experimental data for one- and two-neutron separation energies, implies that among the halo nuclei candidates, only ${ }^{20} \mathrm{C}$ has a possible Efimov state, with an estimated energy less than $14 \mathrm{KeV}$ below the scattering threshold. [S0556-2813(97)50611-X]

PACS number(s): 21.10.Dr, 03.65.Ge, 21.45.+v, 27.20. $+\mathrm{n}$
\end{abstract}

The Efimov effect [1] is the remarkable theoretical observation that the number of bound states for three particles interacting via $s$-wave short-range potentials may grow to infinity, as the pair interactions are just about to bind two particles. Such Efimov states are loosely bound and their wave functions extend far beyond those of normal states. If such states exist in nature they will dominate the low-energy scattering of one of the particles with the bound state of the remaining two particles. Such states have been studied in several numerical model calculations [2-5]. There were searches for Efimov states in atomic and nuclear systems without a clear experimental evidence of occurrence of such states $[6,7]$.

The discovery of halo nuclei brought a lot of attention to the search for Efimov states, because such systems can be viewed as a three-body system with two loosely bound neutrons and a core which is more bound [8-10]. Fedorov, Jensen, and Riisager [5], in a first calculation of possible Efimov states in halo nuclei, suggested as promising candidates the nuclei ${ }^{18} \mathrm{C}$ and ${ }^{20} \mathrm{C}$. They also suggest other possible occurrence in neutron rich oxygen isotopes.

The light three-body halo nuclei have unusual properties in respect to the nuclear size; the radius of the neutron halo is much greater than the radius of the core, and the core is assumed structureless $[4,5,11]$. For the moment, we use this assumption and later on, we discuss its validity in our calculation. This situation allows the use of concepts coming from short-range interactions.

In this work, we settle the general basis for the existence of Efimov states, through the use of universal properties of three-body systems at low energies, in a way convenient to analyze weakly bound systems like the halo nuclei. We have considered that the core has spin zero. The approach is parametrized by the two- and three-body energies in a zerorange model. The renormalizability of the quantum mechanical many-body model with the $s$-wave zero-range force, implies that all the low-energy properties of the three-body system are well defined if one three-body and the low-energy two-body physical informations are known [12]. The threebody input can be chosen as the experimental ground state binding energy. All the detailed informations about the shortrange force, beyond the low-energy two-body observables, are retained in only one three-body physical information in the limit of zero-range interaction. The sensibility of the three-body binding energy to the interaction properties comes from the collapse of the system in the limit of zerorange force, the Thomas effect [13].

The domain of the coupling constants to guarantee threeor four-body bound states, when the subsystems are unbound, was studied in Ref. [14], using short-range interactions. In our approach instead of the strength of the interaction, a quantity not directly available, we fix the energies of the subsystems (bound or virtual) and the three-body ground state by the available experimental data and look for the excited states.

As the input energies are fixed in the renormalized model, a more realistic potential will not affect the generality of the present conclusions. In that sense, the Pauli principle correction, between the halo and the core neutrons, affects essentially the ground state energy which is already fixed. We have to consider that this is a short-range phenomenon that occurs for distances less than the core size (about $\approx 3 \mathrm{fm}$ for light halo nuclei). Considering that we give as input the experimental energies of the ground state three-body system and the subsystems, the spin effect is only roughly considered by our procedure. Our results are strictly valid in the case where the spin of the core is zero. Also, theoretical evidence was found in the last reference of [4], that the conditions for the appearance of the Efimov states become more restrictive for a finite core spin.

The notation used is appropriate for halo nuclei, $n$ for neutron and $c$ for core, but our approach is applicable to any three-particle system which interact via $s$-wave short-range interactions, where two of the particles are identical. The $s$-wave interaction for the $n-c$ potential is justified because the excited state (if exists) should have an extremely small energy, just allowing zero angular momentum for the twoparticle state in the relative coordinates [4]. It was already observed in Ref. [15], when discussing ${ }^{11} \mathrm{Li}$, that even the three-body wave function with an $s$-wave $n$ - $n$ correlation produces a ground state of the halo nuclei with two or more shell-model configurations.

The energies of the two particle subsystem, $E_{n n}$ and $E_{n c}$ can be virtual or bound. In a plane defined by these two 
observables, for example, the ${ }^{11} \mathrm{Li}$ will be represented by a point given by the well-known virtual state of the neutronneutron subsystem $(143 \mathrm{KeV})$, and the not so welldetermined low-energy virtual state of ${ }^{10} \mathrm{Li}(\sim 50 \mathrm{KeV}$, according to [15]). We can vary the mass of the core to hold several other low masses halo nuclei, like ${ }^{11} \mathrm{Li},{ }^{12} \mathrm{Be},{ }^{18} \mathrm{C}$, and ${ }^{20} \mathrm{C}$.

We can anticipate the qualitative behavior of the first Efimov excited state in the halo nuclei by changing the $n-n$ and $n-c$ energies. The Efimov states should disappear in the twobody threshold with the increase of the two-body binding energy [3]. Another result obtained in a previous study shows that the variation of the three-body energy around a vanishing two-body energy is proportional to the square of the absolute value of the two-body bound or virtual state energy [16]. The three-body energy increases with the twobody bound state energy and decreases in the direction of a two-body virtual state. As we will see in the following, this corresponds to weaken the kernel of three-body zero-range equations for the halo nuclei. Comparing with Ref. [14], in our discussion the weakening of the strength is simulated by increasing the two-body virtual state energy.

The zero-range three-body integral equation, for the bound state of two identical particles and a core with spin zero, is written as a generalization of the three-boson equation [17]. It is composed by two coupled integral equations close to the $s$-wave separable potential model of Ref. [11]. The antisymmetrization of the two outer neutrons is satisfied since the spin couples to zero [4]. In our approach the potential form factors and corresponding strengths are replaced, in the renormalization procedure, by the two-body binding energies, $E_{n n}$ and $E_{n c}$. In the case of bound systems, these quantities are the separation energies. We distinguish these two cases by the following definition: $K_{n n} \equiv \pm \sqrt{E_{n n}}$, $K_{n c} \equiv \pm \sqrt{E_{n c}}$, where + refers to bound and - to virtual state energies. Our units will be such that $\hbar=1$ and nucleon mass $=1$.

After partial wave projection, the $s$-wave $n-n-c$ coupled integral equations are

$$
\begin{aligned}
\chi_{n n}(q)= & \tau_{n n}\left(q ; B_{N}\right) \int_{0}^{\Lambda} d k G_{1}\left(q, k ; B_{N}\right) \chi_{n c}(k), \\
\chi_{n c}(q)= & \tau_{n c}\left(q ; B_{N}\right) \int_{0}^{\Lambda} d k\left[G_{1}\left(k, q ; B_{N}\right) \chi_{n n}(k)\right. \\
& \left.+A G_{2}\left(q, k ; B_{N}\right) \chi_{n c}(k)\right],
\end{aligned}
$$

where

$$
\begin{gathered}
\tau_{n n}(q ; E)=\frac{2}{\pi}\left(\sqrt{E+\frac{A+2}{4 A} q^{2}}-K_{n n}\right)^{-1} \\
\tau_{n c}(q ; E)=\frac{1}{\pi}\left(\frac{A+1}{2 A}\right)^{3 / 2}\left(\sqrt{E+\frac{A+2}{2(A+1)} q^{2}}-K_{n c}\right)^{-1}, \\
G_{1}(q, k ; E)=\log \frac{2 A\left(E+k^{2}+q k\right)+q^{2}(A+1)}{2 A\left(E+k^{2}-q k\right)+q^{2}(A+1)}
\end{gathered}
$$

TABLE I. First binding energies, in nondimensional units as defined in the text, as a function of the core mass number $(A)$ of the collapsed three-body system.

\begin{tabular}{lcccccc}
\hline \hline$A=$ & 1 & 5 & 9 & 18 & 50 & 100 \\
\hline $10^{2} \epsilon_{0}=$ & 3.17 & 4.83 & 5.17 & 5.41 & 5.58 & 5.68 \\
$10^{4} \epsilon_{1}=$ & 0.60 & 2.31 & 2.93 & 3.46 & 3.88 & 4.15 \\
$10^{6} \epsilon_{2}=$ & 0.11 & 1.16 & 1.77 & 2.38 & 2.92 & 3.29 \\
\hline \hline
\end{tabular}

$$
G_{2}(q, k ; E)=\log \frac{2(A E+q k)+\left(q^{2}+k^{2}\right)(A+1)}{2(A E-q k)+\left(q^{2}+k^{2}\right)(A+1)} .
$$

$B_{N}$ is the $N$ th three-body halo state energy and $A$ is the core mass number. The cutoff, $\Lambda$, represents the inverse of the interaction radius [7] and it goes to infinity as the radius of the interaction decreases. We made the assumption that the range of $n-n$ and $n-c$ potentials are about the same, represented by just one radius. This assumption should not be regarded as a limitation, since the three-body model is renormalized, requiring only one three-body observable to be fixed [12], together with the two-body low-energy physical informations. The results are independent of the regularization scheme, which in this case means different cutoffs.

The Thomas collapse of the three-body binding energy is seen for $\Lambda \rightarrow \infty$ in Eqs. (1) and (2). This limit is equivalent to the situation that allows the Efimov states, once Thomas and Efimov states are related by a scale transformation [18]. To illustrate this connection, in this particular case, we can make a transformation of Eqs. (1)-(6) to the units in which the cutoff is one. The corresponding equations are formally the same as given in Eqs. (1)-(6), with the dimensional variables and observables replaced by the corresponding nondimensional quantities, such that $\Lambda \rightarrow 1, \quad B_{N} \rightarrow \epsilon_{N} \equiv B_{N} / \Lambda^{2}$, $K_{n n} \rightarrow \kappa_{n n} \equiv K_{n n} / \Lambda, K_{n c} \rightarrow \kappa_{n c} \equiv K_{n c} / \Lambda$. The two-body observables can be written in terms of the three-body energy $B_{N}$, by replacing $\Lambda$, such that $\kappa_{n n} / \sqrt{\epsilon_{N}}=K_{n n} / \sqrt{B_{N}}$ and $\kappa_{n c} / \sqrt{\epsilon_{N}}=K_{n c} / \sqrt{B_{N}}$. The collapse of the three-body energy and the presence of Efimov states are consequences of the existence of solutions of the corresponding equations in the limit of $\kappa_{n n} \rightarrow 0$ and $\kappa_{n c} \rightarrow 0$. The Thomas effect is seen for $\Lambda \rightarrow \infty$ with the energies of the two-body systems fixed, and the Efimov states are seen for $K_{n n} \rightarrow 0$ with $K_{n c} \rightarrow 0$ and $\Lambda$ fixed.

The three-body halo energy scales with the cutoff parameter and the Thomas collapse is seen by the unbound increasing of the energies of the bound states for $\Lambda \rightarrow \infty$. The values of $\epsilon_{N}$ in this limit for the first three states are given in Table I. The excited state energies in the units where $\Lambda=1$ approaches zero by increasing $N$.

In Fig. 1 we have our main results. We show the parametric plane defined by $K_{n c} / \sqrt{B_{N}}$ versus $K_{n n} / \sqrt{B_{N}}$. The curves represent the boundary of the region where there is an excited $(N+1)$ th state above the $N$ th state, for $A=1,9,18$, and 100 . The boundary curve means that the $(N+1)$ three-body binding energy is equal to the lowest scattering threshold. Outside such region the excited bound $(N+1)$ state does not exist. We found that the boundary is practically the same for $N=0$ and $N=1$. The limiting boundary, for $N \rightarrow \infty$, corresponds to the renormalized result in the limit of the zero- 


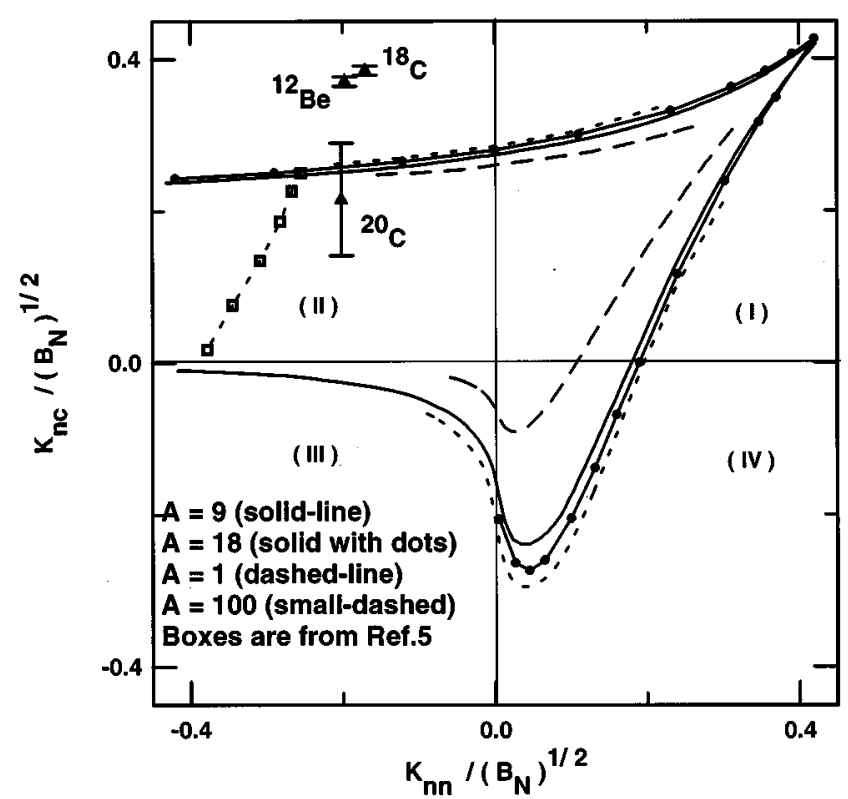

FIG. 1. $K_{n n} / \sqrt{B_{N}}$ as a function of $K_{n c} / \sqrt{B_{N}}$ at the boundary of the region where the binding energy of the $(N+1)$ th Efimov state is zero. Negative values for the two-body observables correspond to virtual states. Boundary curves for different core masses $(A)$; solid line for $A=9$; line with black dots for $A=18$; small dashed line for $A=100$ and dashed line for $A=1$. We also show three experimental data, corresponding to the halo nuclei ${ }^{20} \mathrm{C},{ }^{18} \mathrm{C}$, and ${ }^{12} \mathrm{Be}$ [19]. The squares, connected with dashed lines, are obtained from Fig. 2 of Ref. [5] for $A=9$.

range force. In each step the $N$ th state plays the role of the ground state and $(N+1)$ th state corresponds to the first excited state on the top of the $N$ th state. The energy scale of the $n$ - $n$-core system is given by $B_{N}$. The fast approach of the limiting boundary with $N$ is due to the numerical value of $\epsilon_{0}$, much smaller than 1 , as seen in Table I.

We show four different regions in Fig. 1, where the free two-body subsystems have a virtual or bound state. In region I, the $n-n$ and $n-c$ subsystems have both bound states, and in such case $\kappa_{n n}$ and $\kappa_{n c}$ are positive. In region II, the $n c$-subsystem is bound and the $n n$-subsystem has a virtual state, such that $K_{n c}>0$ and $K_{n n}<0$. The region (III), where $K_{n n}<0$ and $K_{n c}<0$, both subsystems $n-n$ and $n-c$ have virtual states. The region (IV) has $K_{n c}<0$ and $K_{n n}>0$.

We observe an asymmetry between regions II and IV, that can be explained due to the fact that we have two interactions of the kind $n-c$ (between the particle $n$ and the core $c$ ), and just one kind of $n-n$ interaction. So, if $n-n$ is virtual and $n-c$ is bound, as in region II, we have two possibilities of two-body bound states with just one possibility of a twobody virtual state. Therefore, we can easily see that the room for a three-body bound state is very much reduced in region IV compared with region II, as in region IV only one-third of the two-body interactions is giving favorable conditions for binding.

Using this plot we can analyze the existence of Efimov states in any three-body system that interacts via attractive short-range potentials, once the three-body ground state energy and the virtual or bound two-body energies are known.

For the halo nuclei, the relevant section of the plot is the

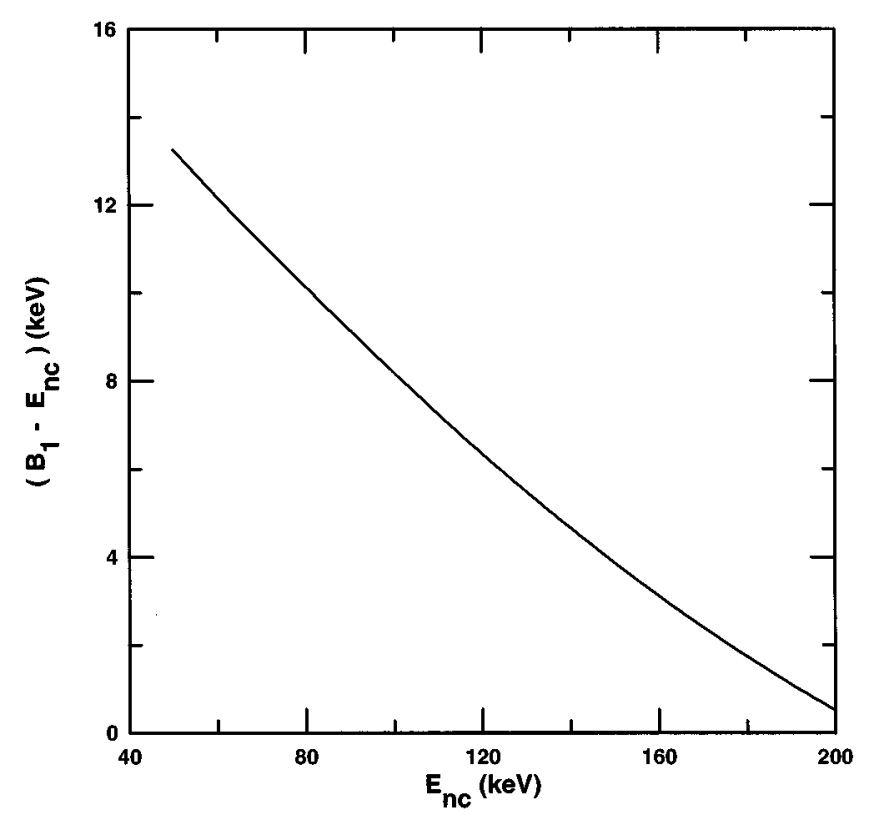

FIG. 2. Binding energy of the ${ }^{20} \mathrm{C}$ Efimov state relative to the elastic threshold as a function of the ${ }^{19} \mathrm{C}$ neutron bound state energy.

regions II and III, because the $n-n$ subsystem has a virtual state $\left(E_{n n}=143 \mathrm{KeV}\right)$. The region we show can accommodate only a few halo nuclei and only the experimental data for ${ }^{20} \mathrm{C}$ [19] is practically inside it the area delimited by the curve that allows Efimov states. Considering the small experimental errors in the data for ${ }^{12} \mathrm{Be},{ }^{18} \mathrm{C}$ [19], we cannot expect to find Efimov states in such nuclei. We are not showing the point corresponding to ${ }^{11} \mathrm{Li}$, because the actual value for the virtual state of ${ }^{10} \mathrm{Li}[15](\sim 50 \mathrm{KeV})$ is ruling out the existence of Efimov states in this nucleus. Only if ${ }^{10} \mathrm{Li}$ had an almost zero virtual energy or being bound with an energy less than $\sim 15 \mathrm{KeV}$, it would be possible an Efimov state. The results for ${ }^{11} \mathrm{Li}$ with a bound ${ }^{10} \mathrm{Li}$ given in Fig. 2 of Ref. [5], are completely inside region II, with an exact agreement of the corresponding threshold.

A brief discussion of the applicability of the results to halo nuclei is needed. The many-body structure of the $N$ th state manifests in the $n$ - $n$-core system, when the two neutrons are inside the core. The three-body dynamics is effective for relative distances bigger than the core size and interaction radius $\left(r_{0}\right)$, if $a_{n n} \gg r_{0}$ and $a_{n c} L \gg r_{0}$ [1,7], with $a_{n n}$ and $a_{n c}$ being the scattering lengths of the $n-n$ and $n$-core subsystems, respectively. Even if the $N$ th state is rather bound, at separation distances where the three-body dynamics is effective the three-body wave function tail is built with the knowledge of the binding energy. The three-body dynamics develops a long-range potential in the hyper-radius of the three-body system [1,7], which carries the informations about $a_{n n}$ and $a_{n c}$. The excited state appears in the longrange potential; it has the same angular components as the ground state, and it is determined by the logarithmic derivative of the ground state wave function at some hyper-radius $\left(r_{b}\right)$, such that $r_{0}<r_{b} \ll a$. This discussion supplies the physical picture of the renormalized three-body model. The values of $a_{n n}$ and $a_{n c}$ for ${ }^{11} \mathrm{Li},{ }^{12} \mathrm{Be},{ }^{18} \mathrm{C}$, and ${ }^{20} \mathrm{C}$ fulfill the condition of being much bigger than the core size and inter- 
action radius. The systematic uncertainties in the application can appear as powers of $r_{0} / a_{n n}$ and $r_{0} / a_{n c}[7](\approx 0.1$ for $\left.{ }^{20} \mathrm{C}\right)$.

The experimental value of the separation energy of the neutron in ${ }^{19} \mathrm{C}$ has a sizable error, $E_{n c}=162 \pm 112 \mathrm{KeV}$ [19], which allows a range for the excited state energy. The experimental value of the three-body ground state energy of ${ }^{20} \mathrm{C}$ has a small error compared to the $E_{n c}$. Thus, we fixed the ground state energy at $3506 \mathrm{KeV}$ [19] and study the excited state energy as a function of $E_{n c}$, as given in Fig. 2. We observe that if $E_{n c}>200 \mathrm{KeV}$, the excited state is destroyed, but in the region of $50 \mathrm{KeV}<E_{n c}<200 \mathrm{KeV}$, the ${ }^{20} \mathrm{C}$ halo nuclei supports the existence of an Efimov state. The binding energy relative to the $n-(n c)$ scattering threshold is below $14 \mathrm{KeV}$. We estimate the size of this excited state to be at least $35 \mathrm{fm}$, supporting its interpretation as an Efimov state. The accuracy of the calculation of the binding energy of the Efimov state will depend on the input experimental energies (the relative error in the binding energy of the neutron in ${ }^{19} \mathrm{C}$, is about 0.7 ) and on the importance of the higher partial waves. Thus, our calculation for ${ }^{20} \mathrm{C}$ relies on the experimental evidence [20] that the last neutron of ${ }^{19} \mathrm{C}$ is in an intruder $s$-orbit.

Actually, the importance of core polarization in halo nuclei has been debated in two recent works [21]. Our method is independent of effects that influence the ground state energy of the system and/or the energies of the subsystems, considering that such energies are given as inputs in the renormalizable three-body model. Although the reference of Kuo, Krmpotić, and Tzeng [21] shows that core polarization is suppressed in halo nuclei, even if it were to be of some residual importance, in our model its effect on the binding of the Efimov state, would be taken into account through the energy of the ground state, which is given as input.

In summary, we have discussed the universal aspects of three-body halo nuclei in the limit of a zero-range interaction. We use the correlation of the value of the ground state energy and the first excited Efimov state, and find the set of values of the $n-n$ and $n-c$ energies that allows the existence of at least one excited Efimov state. We have considered that the core has spin zero and our results in the present form gives an estimate of the energy of the excited state within the renormalized zero-range model. Considering the available data, we conclude that ${ }^{20} \mathrm{C}$ is the strongest candidate for having one excited Efimov state, with an estimated binding energy below $14 \mathrm{KeV}$ relative to the lowest scattering threshold. Our calculation and the available data exclude the possibility of having Efimov states any other light halo nuclei known to us, like ${ }^{11} \mathrm{Li},{ }^{12} \mathrm{Be},{ }^{18} \mathrm{C}$, or oxygen isotopes.

Our thanks for support from Fundação de Amparo à Pesquisa do Estado de São Paulo (FAPESP) and from Conselho Nacional de Desenvolvimento Científico e Tecnológico (CNPq) of Brazil.
[1] V. Efimov, Phys. Lett. 33B, 563 (1970).

[2] A. T. Stelbovics and L. R. Dodd, Phys. Lett. 39B, 450 (1972); A. C. Antunes, V. L. Baltar, and E. M. Ferreira, Nucl. Phys. A265, 365 (1976).

[3] S. K. Adhikari and L. Tomio, Phys. Rev. C 26, 83 (1982); S. K. Adhikari, A. C. Fonseca, and L. Tomio, ibid. 26, 77 (1982).

[4] D. V. Fedorov and A. S. Jensen, Phys. Rev. Lett. 25, 4103 (1993); D. V. Fedorov, E. Garrido, and A. S. Jensen, Phys. Rev. C 51, 3052 (1995).

[5] D. V. Fedorov, A. S. Jensen, and K. Riisager, Phys. Rev. Lett. 73, 2817 (1994).

[6] T. K. Lim, Sr. K. Duffy, and W. C. Damert, Phys. Rev. Lett. 38, 341 (1977); H. S. Huber, T. K. Lim, and D. H. Feng, Phys. Rev. C 18, 1534 (1978).

[7] V. Efimov, Comments Nucl. Part. Phys. 19, 271 (1990), and references therein.

[8] I. Tanihata, J. Phys. G 22, 157 (1996).

[9] C. A. Bertulani, L. F. Canto, and M. S. Hussein, Phys. Rep. 226, 281 (1993).

[10] P. G. Hansen, A. S. Jensen, and B. Jonson, Annu. Rev. Nucl. Part. Sci. 45, 591 (1995); M. V. Zhukov, B. V. Danilin, D. V. Fedorov, J. M. Bang, I. J. Thompson, and J. S. Vaagen, Phys. Rep. 231, 151 (1993).
[11] S. Dasgupta, I. Mazumdar, and V. S. Bhasin, Phys. Rev. C 50, R550 (1994).

[12] S. K. Adhikari, T. Frederico, and I. D. Goldman, Phys. Rev. Lett. 74, 487 (1995); S. K. Adhikari and T. Frederico, ibid. 74, 4572 (1995).

[13] L. H. Thomas, Phys. Rev. 47, 903 (1935).

[14] J.-M. Richard and S. Fleck, Phys. Rev. Lett. 73, 1464 (1994).

[15] M. Zinser et al., Phys. Rev. Lett. 75, 1719 (1995).

[16] A. E. A. Amorim, L. Tomio, and T. Frederico, Phys. Rev. C 46, 2224 (1992).

[17] G. V. Skornyakov and K. A. Ter-Martirosian, Sov. Phys. JETP 4, 648 (1957).

[18] S. K. Adhikari, A. Delfino, T. Frederico, I. D. Goldman, and L. Tomio, Phys. Rev. A 37, 3666 (1988).

[19] G. Audi and A. H. Wapstra, Nucl. Phys. A595, 409 (1995); experimental values for the separation energies obtained via $\mathrm{ftp}$, as suggested in Ref. [1] of this reference.

[20] D. Bazin et al., Phys. Rev. Lett. 74, 3569 (1995).

[21] T. T. S. Kuo, F. Krmpotić, and Y. Tzeng, Phys. Rev. Lett. 78, 2708 (1997); A. Csótó, "Importance of core polarization in halo nuclei,"' nucl-th 9704054. 